\title{
Comparative Analysis of Gaussian Filter with Wavelet Denoising for Various Noises Present in Images
}

\author{
Amanjot Singh ${ }^{1,2 *}$ and Jagroop Singh ${ }^{3}$ \\ 'I.K.G. P.T.U., Jalandhar - 144603,Punjab, India; \\ ${ }^{2}$ School of Electronics and Electrical Engineering, Lovely Professional University, Phagwara - 144411, Punjab, India; \\ er.ajotsingh@gmail.com \\ ${ }^{3}$ Department of Electronics and Communication Engineering, DAVIET, Jalandhar - 144008, Punjab, India; \\ roopasidhu@yahoo.com
}

\begin{abstract}
Objectives: This paper is providing a comparative performance analysis of wavelet denoising with Gaussian filter applied on images contaminated with various noises. Gaussian filter is a basic filter used in image processing. Its response is varying with its kernel sizes that have also been shown in analysis. Wavelet based de-noising is also one of the way of removing various noises usually present in images. Wavelet transform is used to convert the images to wavelet domain. Based on thresholding operations in wavelet domain noise could be removed from images. Methods/Analysis: In this paper, image quality matrices like PSNR and MSE have been compared for the various types of noises in images for different denoising methods. Moreover, the behavior of different methods for image denoising have been graphically shown in paper with MATLAB based simulations. Findings: In the end wavelet based de-noising methods has been compared with Gaussian based filter. The paper provides a review of filters and their denoising analysis under different noise conditions.
\end{abstract}

Keywords: Denoising, Gaussian Filter, MSE, PSNR, SNR, Thresholding, Wavelet Transform

\section{Introduction}

In today's time one massive part of digital data consists of images. In acquisition or transmission of images, these often get corrupted by noises. Denoising of images is a fundamental part of digital image processing which focus on removal of noise while retaining important features. In literature various methods of denoising are present i.e. traditional methods focus on linear techniques and recent on non-linear techniques. There are many image acquisition methods which introduce the various types of noises and artifacts. These noisy parts introduce the undesired visual effects. Therefore, Image denoising is having a significant position in digital image processing.

Gaussian filter is a smoothing filter which has the capability of removing noise and even certain details. Primarily it is a very basic type of filter. It is having a kernel operator which represents the shape of a filter mainly is of bell shaped. Mathematical representation of Gaussian distribution ${ }^{1}$ is also used to represent the Gaussian filter. As per literature it is one the very useful filter of digital image processing $\frac{5,6}{6}$. Its action of filtering can be varied with window size given by the kernel. Results based on variations of kernel parameter have also been represented in the filter.

There are various significant noise removal techniques for images which are based on wavelet thresholding or shrinkage ${ }^{22}$. Wavelet thresholding is one of the nonlinear techniques. It is basic technique as it is based on selection of wavelet coefficients based on specific threshold. Donoho and Johnstone ${ }^{\underline{23}}$ have worked on various wavelet thresholding and shrinkage schemes for denoising 22 . Wavelet approach is one of the successful techniques for removal of noise as followed in literature in last decades. It has been observed that the use of wavelets effectively removes noise while retaining the signal properties,

${ }^{*}$ Author for correspondence 
regardless of variation in frequency content $\underline{23}$. In this paper Section 1 is dealing with Introduction to de-noising of images, Section 2 is dealing with details of Gaussian filter, Section 3 with wavelet based denoising, Section 4 is providing the noise related information and Section 5 is MATLAB based analysis and at last in Section 6 conclusion has been presented .

\section{Gaussian De-Noising}

\subsection{Filter Distribution Structure}

Gaussian filter can be of two types which could base upon its mathematical distribution in 1 dimension or two dimension $\stackrel{8.9}{ }$. Mathematical operators used for representation are as below.

\subsubsection{Gaussian Distribution One Dimensional}

1-D Gaussian distribution has the mathematical form as below:

$$
G(x, y)=\frac{1}{\sqrt{2 \pi} \sigma} e^{-\frac{x^{2}}{2 \sigma^{2}}}
$$

$\sigma$ is the standard deviation for distribution function for Gaussian Filter. If the mean is zero centered at $\mathrm{x}=0$; Distribution is as per Figure 1.

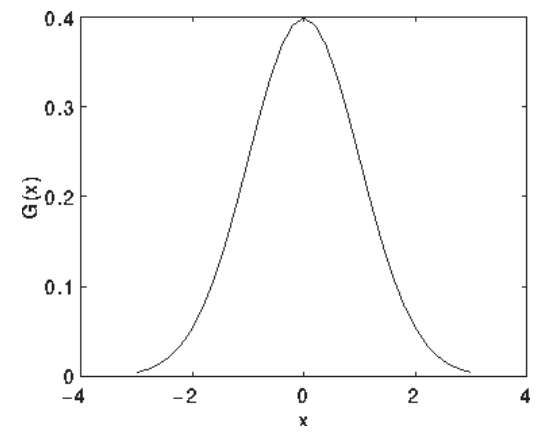

Figure 1. Gaussian distribution in 1D.

\subsubsection{Gaussian Distribution Two Dimensional}

Gaussian distribution in two dimensions is given by:

$$
G(x, y)=\frac{1}{2 \pi \sigma^{2}} e^{-\frac{x^{2}+y^{2}}{2 \sigma^{2}}}
$$

Distribution at $\sigma=1$ and with mean $(0,0)$ (Figure 2).

\subsection{Point Spread Function}

In order to get the Gaussian smoothing point spread function need to be convolved with the image. Image is always represented by the collection of discrete pixels. So the Gaussian filter must be discrete for performing the convolution. Gaussian distribution is non-zero everywhere that leads to infinite large kernel however in practical cases it is made limited as per applications ${ }^{2}$. Further mask is developed approximately as per the Gaussian distribution. Moreover, with suitable selection of kernel standard convolution can be performed for Gaussian smoothing ${ }^{3,10,12}$. Convolution is performed firstly in one direction and then performed in other direction. The 2D-Convolution can be performed in $\mathrm{x}$ and $\mathrm{y}$ direction. Firstly, 1D convolution is performed in $\mathrm{x}$ direction and then convolution is done with y direction.

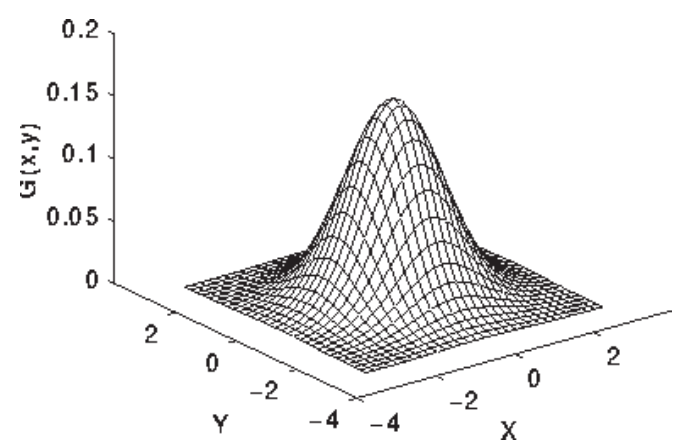

Figure 2. Gaussian distribution in 2D.

\subsection{Gaussian Filtering}

Gaussian filtering mainly results in blurring of an image. In many cases its action is similar to the mean filter. However, the level of smoothing depends upon the Gaussian function's standard deviation. Moreover, its kernel with standard deviation can control the action of Gaussian filtering. The processed image shows the response of Gaussian filter as the weighted average comparatively towards the central pixels. It has been shown that it could give the gentle smoothing and better edges as compared to the mean filter with same size $\frac{1,7}{\text {. }}$.

\section{Wavelet Denoising}

Other way of denoising the images is to transform the domain and removing the undesired effects in other domain. Methods used for it is thresholding i.e. soft and hard thresholding, discussed in further part. 


\subsection{Wavelet Transform}

Wavelet transform based denoising approach is one of the successful techniques for removal of noises in images. It is being observed in the literature that the use of wavelets effectively removes noise without effecting the signal characteristics, despite of variation in frequency content $\frac{16}{}$. Wavelet transformation is technique which decomposes the signal or the image into multi-scale resolutions i.e. There exists a multi-resolution representation of signal or image in two different domains. The localization of wavelet basis functions in the domain of both time and frequency makes the multi-resolution analysis possible. An effective filter design for specific application is possible with wavelet transform. Edge and sharp information is well maintained in wavelet decomposition, so it leads to the very accurate results in edge detection. Moreover, due to these properties it is very useful in image denoising and in related applications. In recent time wavelet based de-nosing have got its applications in many fields like biomedical, multimedia, satellite imaging etc. There are many wavelet functions like haar, symlet, daubechies, biorthogonal and discrete meyer coiflets which have been used for denoising $\underline{18}$.

DWT converts the signal into wavelet domain having number of coefficients where signal energy concentrate. So a noisy image is having multiple coefficients with high SNR (signal to noise ratio) and low SNR. Coefficients with low SNR can be filtered out and high SNR coefficients can be retained. The image can be reconstructed with inverse wavelet transform. So image can be restored to its original position17. The DWT is based on the hierarchy of sub bands spaced in frequency. It is represented by octaveband decomposition. DWT decomposes the image into four sub-bands which is implemented by separate implementation of horizontal and vertical filters. Coefficients with finest scale are knowns as LH1, HL1 and HH1 and coarse level coefficients are known as LL1.This LL1 subband can be further decomposed to next coarse level of coefficients which would lead to next two level wavelet decomposition $\frac{16}{}$.

\subsection{Wavelet based Thresholding}

Wavelet thresholding is useful in signal denoising in order to select the desired DWT coefficients. It is removes the noise be removing the coefficients relevant to specific threshold value. There are specific methods for selecting the threshold values. The method used for it is commonly called as Wavelet Shrinkage. Thresholding Methods can be categorized as hard or soft thresholding which are used for Wavelet based image denoising ${ }^{19}$. Hard and soft thresholding techniques have their own implementation rules. Hard thresholding is implemented on keep or kill rule , However introduces the artifacts while Soft thresholding is implemented on shrink and kill rule. Soft thresholding reduces the abrupt sharp changes and provides a more visually pleasant image Therefore in most of applications soft thresholding is preferred ${ }^{20}$. Mathematical representation of hard and soft thresholding is as per below.

\subsubsection{The Hard Thresholding Function}

$$
\begin{aligned}
& \mathrm{O}(\mathrm{M}, \mathrm{y})=\mathrm{M} \text { for all }|\mathrm{M}|>\mathrm{y} \\
& =0 \text { otherwise }
\end{aligned}
$$

\subsubsection{The Soft Thresholding Function}

$$
\mathrm{O}(\mathrm{M}, \mathrm{y})=\operatorname{sgn}(\mathrm{M})^{\star} \max (0,|\mathrm{M}|-\mathrm{y})
$$

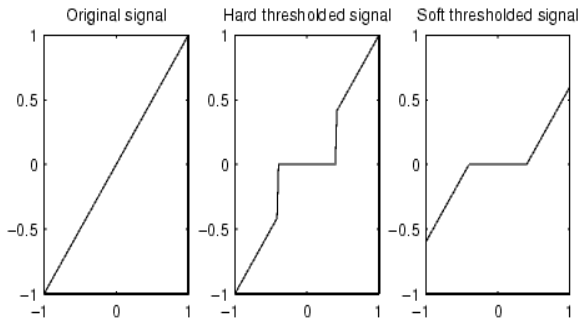

Figure 3. Thresholding characteristics.

\subsection{Threshold Selection Rules}

In order to remove the noise a proper threshold value need to be selected? ${ }^{9}$. Selection of threshold is a challenging task. As if lower value of threshold is there it will pass all noisy components or if higher selected it will remove desired coefficients which could lead to loss of desired components results in artifacts $\frac{16}{}$. There are various threshold selection procedures which have their own pros and cons. Universal Threshold is implemented if number of samples are more. Moreover, the universal threshold provides better estimate for Soft thresholding. Visu Shrink was introduced and implemented by Donoho. It is implemented on hard threshold rule. One of the limitation of shrinkage is that neither speckle noise can be removed nor MSE can be reduced. It gives its good performance with additive noise. The method called as Bayes Shrink is used in setting the different thresholds with every sub 
band. Subbands indicate the bands in frequencies which are different in level and direction from each other ${ }^{16}$. Soft thresholding is used in Bayes Shrink.

\section{Noise Types}

For the performance analysis and to compare the performance of other filters common noise models have been selected. Noise could be of any type practically and noise is also completely unpredictable ${ }^{\frac{14}{4}}$. Various types of noises like Gaussian noise, Salt and pepper noise, Speckle noise, Poisson noise have been considered in order to compare the functioning of different type denoising methods $s$ 11,13. Further parts of the paper shows the graphical comparisons of filters in terms of PSNR and MSE with these models. The various types of noises have their own different characteristics which have been discussed further.

\subsection{Salt and Pepper Noise}

Salt and pepper type of impulsive noise typically seen on images. It is mainly indicated as randomly occurring white and black pixels in images. Salt and pepper noise comes into images in situations where fast transients take place. Salt and Pepper is also known as intensity spikes which are generally caused due to errors in transmission. It exists in two possible values i.e. black or white. The probability of occurrence of each spot is very small. The corrupted pixels are observed as Salt and Pepper like appearance alternatively to minimum or to maximum values. The unaffected pixels remain in usual values $\frac{11,18}{}$.

\subsection{Gaussian Noise}

Gaussian Noise is a noise which is following the Gaussian distribution. It is randomly distributed over the image. One of its special case called as the white Gaussian noise which is completely statistically uncorrelated. Noisy image contains the sum of true pixel values as well as random Gaussian distributed noise value for each noisy pixel ${ }^{18}$.This type of noise is completely unpredictable . Mostly it is present in old images or due to blurring of images.

\subsection{Speckle Noise}

It is a multiplicative noise $\mathrm{e}^{21}$. Speckle noise occurs in images like ultrasound images, SAR images. So its existence is mainly in medical images. It mainly originates from the backscatter waves. Microscopic diffused reflections in internal organs make the backscatter waves. Speckle noise is having the gamma distribution ${ }^{5}$. In medical image processing removal of such a type of noise is essential.

\subsection{Poisson Noise}

Poisson noise is image Data dependent. Non-linear response of the recorders and detectors introduces the Poisson noise. Random electron emission is having a Poisson distribution which is involved in detection and recordings. It is considered that noise is having unity variance $\frac{18}{}$.

\section{Simulations and Analysis}

In order to give the comparative analysis firstly performance of Gaussian filtering has been shown, then performance of wavelet based denoising has been analyzed. Finally performance of these two have also been compared with different noises based on quality matrices PSNR (Peak signal to noise ratio) as well as with MSE (Mean square error). The 'child.bmp' (512 X 512) has been taken as a test image. Various types of noises have been added to it as shown in figures. MATLAB software has been used to simulate the complete system and for implementing the Gaussian filter as well as Wavelet thresholding.

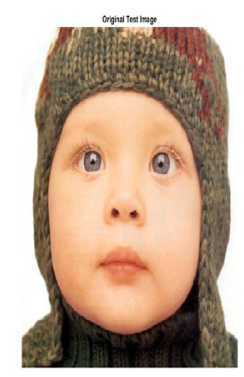

Figure 4. Original Test image 'child.bmp' (512 X 512). 


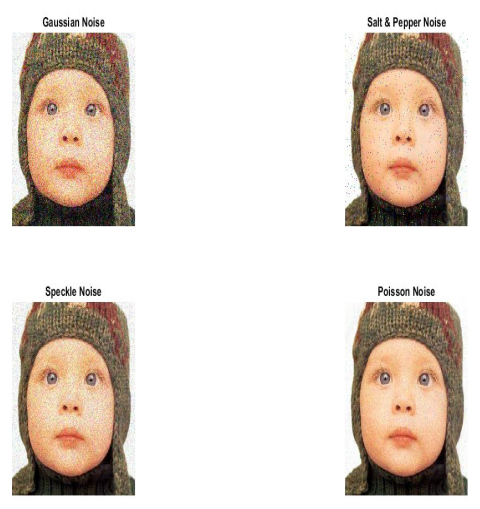

Figure 5. Noisy images.

\subsection{Gaussian Filter Performance}

Gaussian filter works behaves differently for the different types of noises. To check the effect of kernel variations on filter performance, test image has been filtered with varying values of Gaussian filter kernel. This has been done for all types of noises. Sigma of Gaussian filter function decides the kernel value. Kernel for the Gaussian operator has been changed with starting from 0.4 to ending at 2. Results have proved that kernel varies the response of denoising.

Image quality matrices PSNR and MSE have shown the response of filter. Results shows that values of kernel between 0.6 to 1 give best results in filtering of test image for all types of noises. PSNR and MSE have been calculated with help of MATLAB for all type of noises and have been shown graphically and in tables.
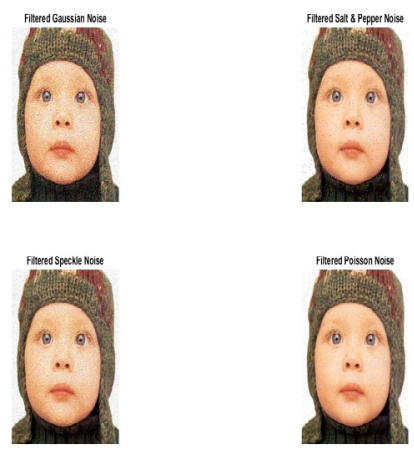

Figure 6. Gaussian filtered images.

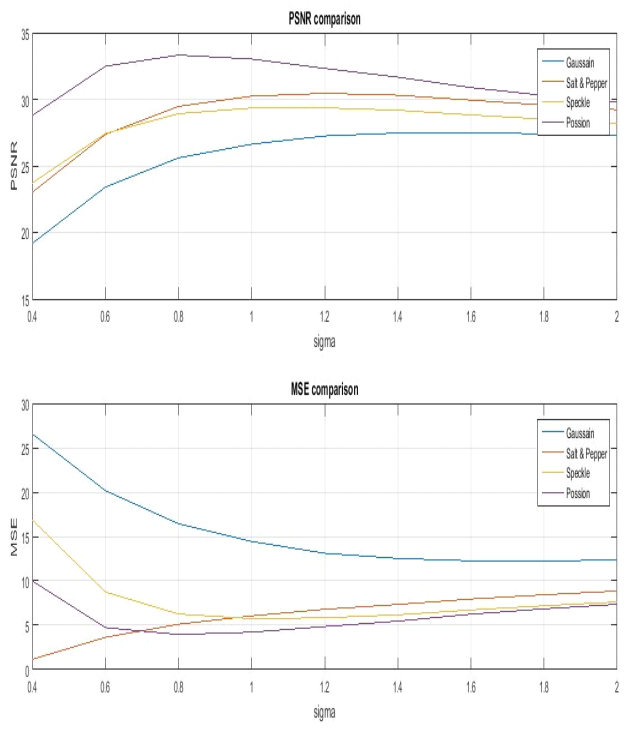

Figure 7. PSNR for different noises with Kernel variations. Table 1. PSNR variations for different noises

\begin{tabular}{|l|l|l|l|l|}
\hline $\begin{array}{l}\text { Kernel } \\
\text { size }\end{array}$ & Gaussian & $\begin{array}{l}\text { Salt \& } \\
\text { Pepper }\end{array}$ & Speckle & Poisson \\
\hline $\mathbf{0 . 4}$ & 19.18499 & 23.02493 & 23.72349 & 28.79324 \\
\hline $\mathbf{0 . 6}$ & 23.41271 & 27.36663 & 27.43494 & 32.48618 \\
\hline $\mathbf{0 . 8}$ & 25.60288 & 29.4693 & 28.92279 & 33.32299 \\
\hline $\mathbf{1}$ & 26.6308 & 30.24109 & 29.34694 & 33.02617 \\
\hline $\mathbf{1 . 2}$ & 27.24739 & 30.44835 & 29.36115 & 32.32083 \\
\hline $\mathbf{1 . 4}$ & 27.46831 & 30.31052 & 29.17768 & 31.665 \\
\hline $\mathbf{1 . 6}$ & 27.4963 & 29.93858 & 28.83016 & 30.88169 \\
\hline $\mathbf{1 . 8}$ & 27.40434 & 29.56811 & 28.50897 & 30.29223 \\
\hline $\mathbf{2}$ & 27.2646 & 29.2178 & 28.21081 & 29.80306 \\
\hline
\end{tabular}

Table 2. MSE variations for different noises

\begin{tabular}{|l|l|l|l|l|}
\hline $\begin{array}{l}\text { Kernel } \\
\text { size }\end{array}$ & Gaussian & $\begin{array}{l}\text { Salt \& } \\
\text { Pepper }\end{array}$ & Speckle & Poisson \\
\hline 0.4 & 26.55627 & 1.165405 & 16.84983 & 9.98346 \\
\hline $\mathbf{0 . 6}$ & 20.16428 & 3.638102 & 8.745274 & 4.737233 \\
\hline $\mathbf{0 . 8}$ & 16.43269 & 5.117533 & 6.263068 & 3.956898 \\
\hline $\mathbf{1}$ & 14.4394 & 6.057225 & 5.746314 & 4.219809 \\
\hline $\mathbf{1 . 2}$ & 13.11074 & 6.80147 & 5.841681 & 4.853913 \\
\hline $\mathbf{1 . 4}$ & 12.52783 & 7.344335 & 6.173839 & 5.466435 \\
\hline $\mathbf{1 . 6}$ & 12.25851 & 7.955143 & 6.723757 & 6.247589 \\
\hline $\mathbf{1 . 8}$ & 12.24698 & 8.43525 & 7.198311 & 6.850081 \\
\hline $\mathbf{2}$ & 12.34604 & 8.857562 & 7.626556 & 7.358636 \\
\hline
\end{tabular}




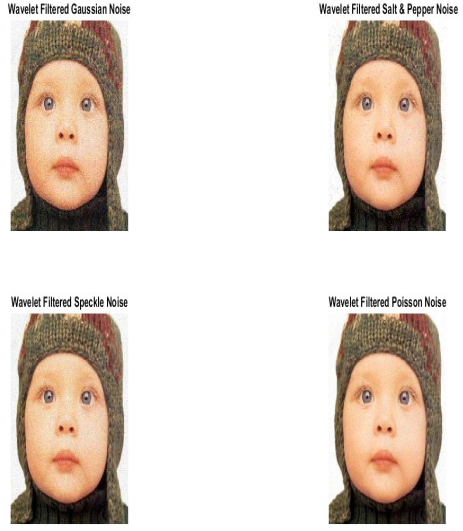

Figure 8. Wavelet denoising with soft thresholding.

It has been observed that certain kernel values are giving better response with respective type of noise. However, it is also providing a good indication that as per application and need its value could be selected for better filter response and making it suitable for noise removal rather than giving blurring effect.

\subsection{Wavelet Thresholding Performance}

Here Thresholding based wavelet denoising method has been considered. It has been implemented in two ways one is hard and other is soft thresholding.

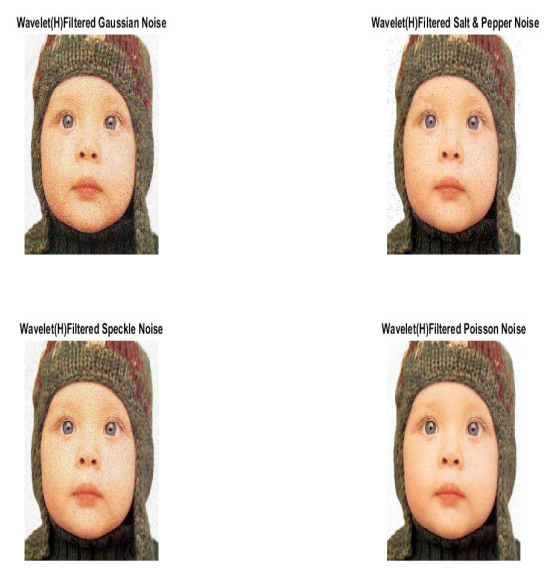

Figure 9. Wavelet denoising with hard thresholding.

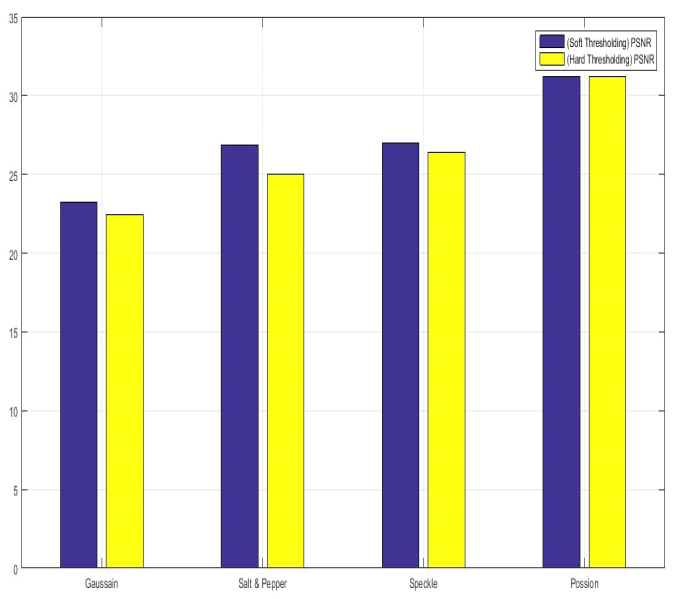

Figure10. Comparison of Hard and soft thresholding PSNR

Table 3. Comparison of hard and soft thresholding (PSNR)

\begin{tabular}{|l|l|l|}
\hline Noise Type & $\begin{array}{l}\text { Soft thresholding } \\
\text { PSNR }\end{array}$ & $\begin{array}{l}\text { Hard thresholding } \\
\text { PSNR }\end{array}$ \\
\hline Gaussian & 23.21863241 & 22.45475 \\
\hline $\begin{array}{l}\text { Salt \& } \\
\text { Pepper }\end{array}$ & 26.88069009 & 25.02254 \\
\hline Speckle & 27.0126296 & 26.39141 \\
\hline Poisson & 31.23782646 & 31.20906 \\
\hline
\end{tabular}

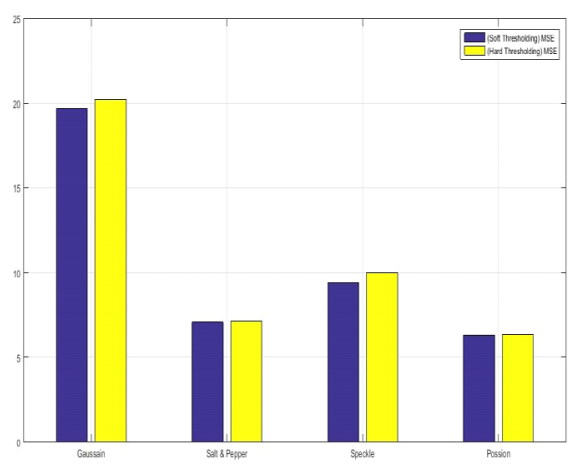

Figure 11. Comparison of hard and soft thresholding (MSE).

Table 4. Comparison of hard and soft thresholding (MSE)

\begin{tabular}{|l|l|l|}
\hline Noise Type & Soft thresholding & Hard thresholding \\
\hline Gaussian & 19.68704 & 20.23421 \\
\hline Salt \& Pepper & 7.067028 & 7.110882 \\
\hline Speckle & 9.411871 & 10.00347 \\
\hline Poisson & 6.298036 & 6.321621 \\
\hline
\end{tabular}




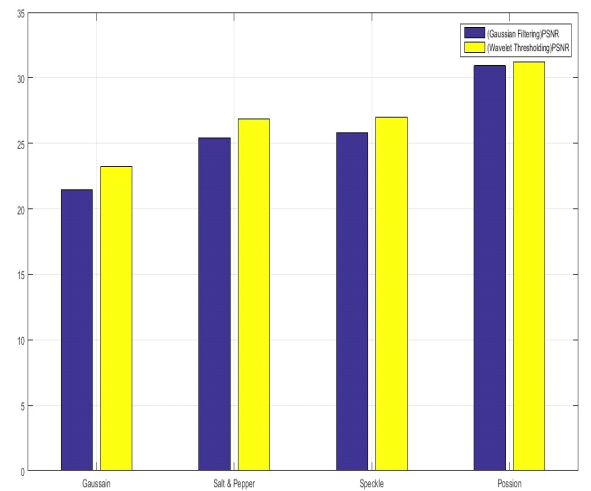

Figure 12. PSNR comparison Gaussian filter with wavelet based denoising.

It has been seen from the PSNR and MSE that Soft thresholding performs the better than the hard thresholding method. Moreover appearance of images with soft thresholding is more smoother. So in wavelet based denoising methods soft thresholding method is preferred .

\subsection{Comparison Gaussian Filter with Wavelet based Denoising}

In this section wavelet and Gaussian filters have been considered for same noises and results are as below. PSNR, SNR and MSE have been compared for the various noises with the two de-noising techniques.

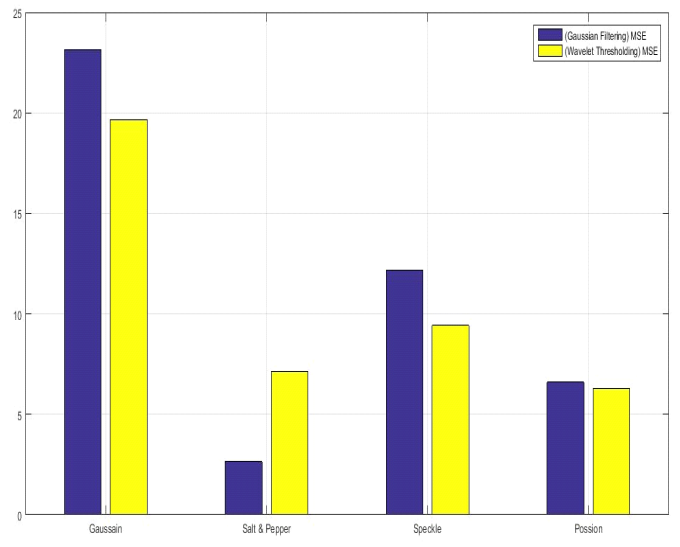

Figure 13. MSE comparison Gaussian filter with wavelet based denoising.

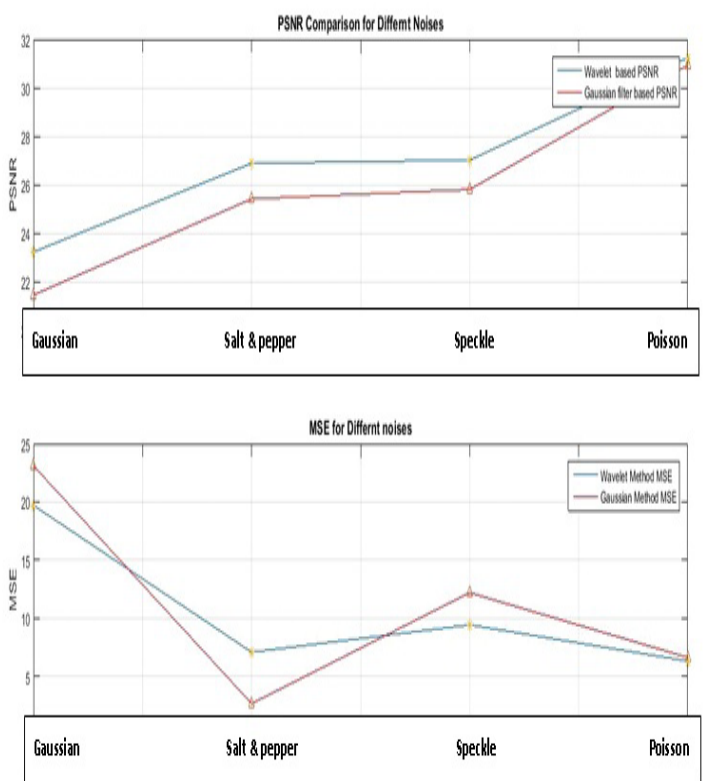

Figure 14. Graphical comparison Gaussian filter with wavelet based denoising (Upper PSNR, Lower MSE).

Table 4. Overall comparison Gaussian filter with wavelet based denoising

\begin{tabular}{|c|c|c|c|c|c|}
\hline & $\begin{array}{l}\text { Noise } \\
\text { type }\end{array}$ & Gaussian & $\begin{array}{l}\text { Salt \& } \\
\text { Pepper }\end{array}$ & Speckle & Poisson \\
\hline \multirow{3}{*}{ 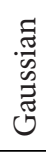 } & PSNR & 21.44457 & 25.40851 & 25.80015 & 30.9263 \\
\hline & MSE & 23.09314 & 2.639663 & 12.18065 & 6.603898 \\
\hline & SNR & 17.23517 & 21.19911 & 21.59075 & 26.7169 \\
\hline \multirow{3}{*}{ 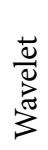 } & PSNR & 23.21863 & 26.88069 & 27.01263 & 31.23783 \\
\hline & MSE & 19.68704 & 7.067028 & 9.411871 & 6.298036 \\
\hline & SNR & 19.00923 & 22.67129 & 22.80323 & 27.02842 \\
\hline
\end{tabular}
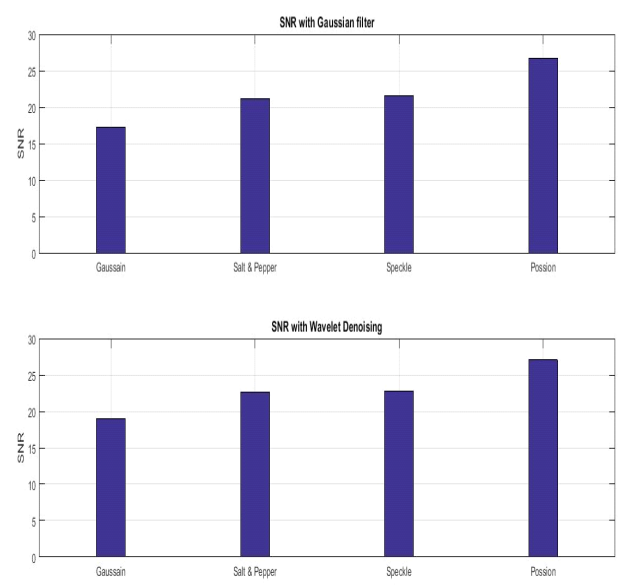

Figure 15. SNR Comparison Gaussian filter (Upper) with Wavelet based denoising (Lower). 
It has been observed that performance of Wavelet based method is better than the Gaussian based methods. PSNR and MSE values have been compared for these different methods of denoising with same noise levels as shown in table. Performance of both methods can be improved with proper selection of various parameters of these filters.

\section{Conclusion}

In this paper performance analysis has been done for Gaussian filter as well as wavelet based filtering, applied on various images contaminated with different noises. It has been seen that there are variations in PSNR and MSE values with different kernel values of Gaussian filter. In the similar way PSNR and MSE have also been varying for the hard and soft thresholding with various types of noises. Based on overall comparison, it has been observed that wavelet based denoising techniques have given the better response as compared to Gaussian filters in terms PSNR as well as MSE for the same type of noises. However Gaussian based filtering can also be improved with proper selection of kernel size. Moreover with proper selection of thresholding method for Wavelet denoising, performance of wavelet based methods for image denoising can also be further improved.

\section{References}

1. Gonzale RC, Woods RE. Digital image processing. Second edition, Upper Saddle River, NJ: Prentice Hall; 2006.

2. Haralick R, Shapiro L. Computer and robot vision. Chapter 7. Addison-Wesley Publishing Company; 1992.

3. Vernon D. Machine vision. Prentice-Hall. 1991; p. $59-61,214$.

4. Gonzale RC, Woods RE. Digital image processing using Matlab. Third edition, Pearson Education; 2005.

5. Pratt WK. Digital image processing. Third edition, A Wiley Inter Science Publication; 2001.

6. Zhou H, Wu J, Zhang J. Digital image processing. Part II. Bookboon; 2010.

7. Jain AK. Fundamentals of digital image processing. Prentice Hall of India; 2002.

8. Sridhar S. Digital image processing. Oxford University Press; 2011.
9. Jähne B. Digital image processing. Sixth edition, Springer; 2005.

10. Zhou H, Wu J, Zhang J. Digital image processing. Part I, Bookboon; 2010.

11. Chan RH, Ho C-W, Nikolova M. Salt -and-pepper noise removal by median -type noise detectors and detail preserving regularization. IEEE Transactions on Image Processing. 2005 Oct; 14(10).

12. Hsiao P-Y, Chou S-S, Huang F-C. Generic 2-D Gaussian smoothing filter for noisy image processing. TENCON 2007 - 2007 IEEE Region 10 Conference; 2007. p. 1-4.

13. Barcelos CAZ, Batista MA. Image restoration using digital inpainting and noise removal. Image and Vision Computing. 2007; 25:61-9.

14. Liu C, Szeliski R, Kang SB, Zitnick CL, Freeman WT. Automatic estimation and removal of noise from a single image. IEEE Transactions on Pattern Analysis and Machine Intelligence. 2008 Feb; 30(2).

15. Mohideen SK, Perumal DA, Sathik MM. Image de-noising using discrete wavelet transform. International Journal of Computer Science and Network Security. 2008 Jan; 8(1).

16. Anutam, Rajni. Performance analysis of image denoising with wavelet thresholding methods for different levels of decomposition. The International Journal of Multimedia and Its Applications. 2014 Jun; 6(3).

17. Ram I, EladM. Generalized tree-based wavelet transform. IEEE Transactions on Signal Processing. 2011 Sep; 59(9).

18. Rupinderpal. Survey of de-noising methods using filters and fast wavelet transform. International Journal of Advanced Research in Computer Science and Software Engineering. 2013 Feb; 3(2):133-6.

19. Mohideen SK, Perumal DA, Sathik MM. Image de-noising using discrete wavelet transform. International Journal of Computer Science and Network Security. 2008 Jan; 8(1).

20. Fathi A, Naghsh-Nilchi AR. Efficient image denoising method based on a new adaptive wavelet packet thresholding function. IEEE Transaction on Image Processing; 2012 Sep; 21(9).

21. Gagnon, L. Wavelet filtering of speckle noise- ome numerical results. Proceedings of the Conference Vision Interface, Trois-Reveres; 1999.

22. Donoho DL. De-noising by soft-thresholding. IEEE Transactions on Information Theory. 1995 May; 41:613-27.

23. Chang SG, Yu B, Vetterli M. Adaptive wavelet thresholding for image denoising and compression. IEEE Transactions on Image Processing. 2000 Sep; 9(9). 